\section{The Effect of Stellate Ganglion Block on Prolonged Post-operative Ocular Pain}

Purpose: There are cases when ocular pain persists long after ocular surgery and cannot be reduced by antiinflammatory drugs. Our purpose was to evaluate the effect of stellate ganglion block (SGB) on such prolonged postoperative ocular pain.

Method: The subjects were 35 patients divided into two groups. One we called the nociceptive pain group, containing 29 cases in which the trigeminal nerve in the orbit or passing through it was intact. The other we called the neuropathic pain group, containing 6 cases in which pain accompanied paralysis of the trigeminal nerve. SGB was performed two times per week. SGB was deemed effective when pain was reduced markedly and the administration of anti-inflammatory drugs became almost unnecessary.

Results: SGB was $96.6 \%$ effective in the nociceptive pain cases with the SGB performed an average of 5.9 times. On the other hand, in the neuropathic pain group, even though SGB was performed an average of 52.6 times it was only $66.7 \%$ effective.

Conclusions: SGB may be useful for the treatment of prolonged post-operative ocular pain, but the elimination of neuropathic pain is more difficult than the elimination of nociceptive pain.

Nippon Ganka Gakkai Zasshi (J Jpn Ophthalmol Soc 107:607-612, 2003)

\footnotetext{
Masaji Matsuura ${ }^{1}$, Masako Matsuura ${ }^{1}$, Fumitaka Ando ${ }^{2}$, Kazuhiro Sahashi ${ }^{3}$, Yoshihiko Torii ${ }^{3}$ and Hiroshi Hirose ${ }^{3}$

${ }^{1}$ Matsuura Eye Clinic; ${ }^{2}$ Eye Care Nagoya $;{ }^{3}$ Department of Ophthalmology, Nagoya National Hospital
}

DOI 10.1007/s10384-004-1023-5

\section{A Case of Optic Nerve Drusen Associated with Grönblad-Strandberg Syndrome}

Purpose: We report a rare case of optic nerve drusen associated with Grönblad-Strandberg syndrome.

Case: The patient was a 29 -year-old woman who had pseudoxanthoma elasticum on her neck.

Observations: On eye examination, there were no abnormal findings in the anterior segment or ocular media. Ophthalmoscopic examination revealed milky white granular lesions on the optic nerve disc, and angioid streaks around the disc in both eyes. Orbital computerized tomography (CT), B-scan echography and photography with a fluorescein angiography filter identified the granular lesions as optic nerve drusen. With a scanning laser ophthalmoscope (SLO), poorly visible drusen buried below the nerve head could be detected. The optic nerve drusen could be seen more clearly by infrared laser than by helium-neon laser.

Conclusions: SLO using infrared laser is useful for evaluation of superficial and buried drusen.

Nippon Ganka Gakkai Zasshi (J Jpn Ophthalmol Soc 107:613-618, 2003)

\section{Ryoko Kumano, Kenji Ibi, Yuko Koketsu and Akihiko Tawara \\ Department of Ophthalmology, University of Occupational and Environmental Health, Japan}

DOI 10.1007/s10384-004-1024-4

These abstracts, which appeared in recent issues of the Journal of the Japanese Ophthalmological Society, have been edited for publication in the Japanese Journal of Ophthalmology. 